\title{
On the Regularity and Chaos of the Hydrogen Atom Subjected to External Fields
}

\author{
Jaouad Kharbach ${ }^{1}$, Walid Chatar ${ }^{1}$, Mohamed Benkhali' ${ }^{1}$ Abdellah Rezzouk ${ }^{1}$, \\ Mohammed Ouazzani-Jamil²
}

\begin{abstract}
${ }^{1}$ Laboratoire de Physique du Solide, Faculté des Sciences Dhar El Mahraz, Université Sidi Mohamed Ben Abdellah, Fès-Atlas, Morocco

${ }^{2}$ Université Privée de Fès, Laboratoire Systèmes et Environnements Durables, Lot. Quaraouiyine Route Ain Chkef, Fès, Morocco

Email: kharbach@gmail.com
\end{abstract}

How to cite this paper: Kharbach, J., Chatar, W., Benkhali, M., Rezzouk, A. and Ouazzani-Jamil, M. (2018) On the Regularity and Chaos of the Hydrogen Atom Subjected to External Fields. International Journal of Modern Nonlinear Theory and Application, 7, 56-76.

https://doi.org/10.4236/ijmnta.2018.72005

Received: April 13, 2018

Accepted: June 26, 2018

Published: June 29, 2018

Copyright $\odot 2018$ by authors and Scientific Research Publishing Inc. This work is licensed under the Creative Commons Attribution International License (CC BY 4.0).

http://creativecommons.org/licenses/by/4.0/

\begin{abstract}
In this paper, the integrable classical case of the Hydrogen atom subjected to three static external fields is investigated. The structuring and evolution of the real phase space are explored. The bifurcation diagram is found and the bifurcations of solutions are discussed. The periodic solutions and their associated periods for singular common-level sets of the first integrals of motion are explicitly described. Numerical investigations are performed for the integrable case by means of Poincaré surfaces of section and comparing them with nearby living nonintegrable solutions, all generic bifurcations that change the structure of the phase space are illustrated; the problem can exhibit regularity-chaos transition over a range of control parameters of system.
\end{abstract}

\section{Keywords}

Hydrogen Atom, Zeeman Effect, Stark Effect, Van Der Waals Interaction, Hamiltonian System, Integrability, Bifurcation, Chaos, Poincaré Surfaces of Section

\section{Introduction}

Most natural phenomena are generally governed by nonlinear differential equations, for these problems, we must use different mathematical approaches and computational methods to simplify these systems and to study their integrable like lie algebra, the Painlevé criterion, the Ziglin criterion, the Liouville theorem and the Poincaré sections, because physically integrable systems are rare.

The study of the Hydrogen atom has been recently the focus of many works [1]-[10]. Its great importance lies in its fields of implications in different 
branches of physics: solid state physics [11], spectroscopy [12], plasmas physics [13], molecular physics [14], astrophysics [15].

The Hydrogen atom is a simple but very complicated system when introducing external fields. The Hydrogen atom in a static magnetic field is a simple system that can be studied experimentally and theoretically, its dynamics in such a field has been a source of many interesting results in atomic physics over the past two decades [16] [17]. It proves to be a prototype of quantum systems whose classical behavior can show chaotic dynamics [18] [19]. Concrete systems are often modeled using systems with natural parameters. The regularity of the behavior of the system is observed for certain values of the parameters, but usually the behavior of the dynamical system becomes unpredictable.

The Hydrogen atom under the effect of external fields is a complicated, non-separable problem and generally displays chaotic behavior for some combinations of the appropriate parameters. The chaotic appearance results from the fact that, for certain initial conditions, the behavior of the dynamic system becomes unpredictable [20]. The chaos is identified by a high sensitivity to the initial conditions and by the variation of the control parameters of the system. Indeed, a system with $\mathrm{n}$ degrees of freedom is regular if there are $\mathrm{n}$ independent constants of motion [21]. Most problems involving atoms in external fields are very difficult to study analytically and solutions are difficult to obtain. However, for combinations of appropriate parameters, one can find exact solutions.

The isotropic nonrelativistic Hydrogen atom subjected to three static external fields: a magnetic field, an electric field, and a van der Waals interaction. The Hamiltonien (in units such that $m_{e}=\hbar=e=a_{0}=1$ ) can be written as:

$$
H=\frac{1}{2} p^{2}-\frac{1}{r}+\alpha\left(x^{2}+y^{2}+\lambda^{2} z^{2}\right)+\gamma\left(x^{2}+y^{2}\right)+\beta z
$$

where $r^{2}=x^{2}+y^{2}+z^{2}, p^{2}=p_{x}^{2}+p_{y}^{2}+p_{z}^{2}$, and $\alpha, \lambda, \gamma$ and $\beta$ are control parameters representing the magnitude of the applied external fields: $\alpha$ is a constant; $\gamma$, measured in units of magnetic field $B_{0}=2.35 \times 10^{5} \mathrm{~T}$, controls the quadratic Zeeman effect; $\beta$, measured in units of electric field $F_{0}=5.14 \times 10^{11} \mathrm{~V} / \mathrm{cm}$, controls the Stark effect; and the a-dimensional number $\lambda$ controls the anharmonicity associated to the van der Waals interaction [10] [11] [12]. This combined potential is very interesting because of its similarity to the fields seen by an ion confined in a Paul trap [13].

The paper is organized as follows: In Section 2 we briefly review the regularized equations of motion and we bring out that the regular 2D Hamiltonian is equivalent to the motion of two coupled anharmonic oscillators. Section 3 presents a detailed description of the real phase space topology on the bifurcation diagram. For noncritical values of the constants of motion $h$ and $f$, the regular level sets of the first integrals of motion of a completely integrable Hamiltonian system are composed of tori, according to the Liouville theorem. All generic bifurcations of Liouville tori, corresponding to critical values of the constants of motion $h$ and $f$ will be described, by using Fomenko's surgery. In Section 4 we give explicit periodic solutions and their associated periods for singular com- 
mon-level sets of the constants of motion. In section 5 we also investigate numerically via Poincaré surfaces of section, the structure and evolution of the phase space for the integrable case, comparing them with nearby living nonintegrable solutions, the problem can exhibit regularity-chaos transition over a range of system control parameters. Finally, Section 6 contains our conclusions.

\section{Equations of Motion}

The regularized Hamiltonian is obtained by transforming Equation (1) to semiparabolic coordinates and the corresponding momenta, namely,

$$
\begin{aligned}
& x=u v \cos (\varphi), \quad p_{x}=\frac{v \cos (\varphi)}{u^{2}+v^{2}} \frac{v \cos (\varphi)}{u^{2}+v^{2}} p_{u}+\frac{u \cos (\varphi)}{u^{2}+v^{2}} p_{v}-\frac{\sin (\varphi)}{u v} p_{\varphi}, \\
& y=u v \sin (\varphi), \quad p_{y}=\frac{v \sin (\varphi)}{u^{2}+v^{2}} \frac{v \sin (\varphi)}{u^{2}+v^{2}} p_{u}+\frac{u \sin (\varphi)}{u^{2}+v^{2}} p_{v}+\frac{\cos (\varphi)}{u v} p_{\varphi}, \\
& z=\frac{u^{2}-v^{2}}{2}, \quad p_{z}=\frac{u}{u^{2}+v^{2}} \frac{u}{u^{2}+v^{2}} p_{u}-\frac{v}{u^{2}+v^{2}} p_{v} .
\end{aligned}
$$

Note that the equations of motion associated with the Hamiltonian (1) have a collision singularity at $r=0$, which can be removed with the following change in the independent variable

$$
\frac{\mathrm{d} t}{\mathrm{~d} \tau}=r=\left(u^{2}+v^{2}\right)
$$

After such transformation, the regularized Hamiltonian reads

$$
\begin{aligned}
\tilde{H}(u, v)= & =\frac{1}{2}\left(p_{u}^{2}+p_{v}^{2}\right)+\frac{p_{\varphi}^{2}}{2}\left(\frac{1}{u^{2}}+\frac{1}{v^{2}}\right)-H\left(u^{2}+v^{2}\right)+\frac{\alpha \lambda^{2}}{4}\left(u^{6}+v^{6}\right) \\
& +\frac{\beta}{2}\left(u^{4}-v^{4}\right)+\left(\alpha+\gamma-\frac{\alpha \lambda^{2}}{4}\right)\left(u^{4} v^{2}+u^{2} v^{4}\right)
\end{aligned}
$$

In this equation, $p_{\varphi}$ is a conserved quantity, represents the value of the cyclic integral associated to the cyclic coordinate $\varphi$, and we put $p_{\varphi}=l$, with $l$ a constant.

The difference between the Hamiltonian (3) and the Hamiltonian of an ion in a Paul trap is that the total pseudo-energy $\tilde{H}$ is equal to 2, that of a trapped ion is -2 (for details, see Ref. [11]).

The equations of motion associated with $\tilde{H}(u, v)$ in the new time $\tau$ are, therefore,

$$
\begin{aligned}
& \dot{u}=p_{u}, \\
& \dot{p}_{u}=2 h u-2 \beta u^{3}-6 \frac{\alpha \lambda^{2}}{4} u^{5}+\frac{l^{2}}{u^{3}}-\left(\alpha+\gamma-\frac{\alpha \lambda^{2}}{4}\right)\left(4 u^{3} v^{2}+2 u v^{4}\right), \\
& \dot{v}=p_{v}, \\
& \dot{p}_{v}=2 h u+2 \beta v^{3}-6 \frac{\alpha \lambda^{2}}{4} v^{5}+\frac{l^{2}}{v^{3}}-\left(\alpha+\gamma-\frac{\alpha \lambda^{2}}{4}\right)\left(4 u^{2} v^{3}+2 u^{4} v\right) .
\end{aligned}
$$

the dot denotes derivative with respect to $\tau$.

The Hamiltonian (3) characterizes a two dimensional regularized version of 
the real three dimensional system after ignoring the cyclic integral associated to the cyclic coordinate $\varphi, p_{\varphi}=l=0$.

The study of the dynamics for Hydrogen atom subjected to external fields is easier to process in two-dimensional standardized version than a three-dimensional; and by removing the Coulomb singularity from the original dynamic system, the regularization procedure facilitates the numerical work. Another practical aspect is that the regular 2D Hamiltonian is equivalent to the motion of two coupled anharmonic oscillators with pseudo-energy is equal to 2, a system for which there are effective tools to make the problem easily exploitable.

\section{The Phase Space Topology}

The two dimensional regularized Hamiltonian (3) is separable if the relation (5) is verified

$$
\alpha+\gamma-\frac{\alpha \lambda^{2}}{4}=0 \Leftrightarrow \lambda= \pm 2 \sqrt{1+\frac{\gamma}{\alpha}}
$$

in this case the second integral of motion reads

$$
\begin{gathered}
F=f=\frac{\left(u^{2} p_{v}^{2}-v^{2} p_{u}^{2}\right)}{2\left(u^{2}+v^{2}\right)}+\frac{(\alpha+\gamma)\left(v^{6} u^{2}-u^{6} v^{2}\right)+2 v^{2}}{u^{2}+v^{2}}-\beta \frac{\left(v^{4} u^{2}+u^{4} v^{2}\right)}{2\left(u^{2}+v^{2}\right)} \\
F=f=z p_{x}^{2}-x p_{x} p_{z}+1-\frac{z}{\sqrt{x^{2}+z^{2}}}-2(\alpha+\gamma) x^{2} z-\frac{\beta}{2} x^{2}
\end{gathered}
$$

It is easy to verify that Equation (2) becomes

$$
\begin{array}{ll}
x=u v, & p_{x}=\frac{v}{u^{2}+v^{2}} \frac{v}{u^{2}+v^{2}} \sqrt{g_{1}(u)}+\frac{u}{u^{2}+v^{2}} \sqrt{g_{2}(v)}, \\
z=\frac{u^{2}-v^{2}}{2}, & p_{z}=\frac{u}{u^{2}+v^{2}} \frac{u}{u^{2}+v^{2}} \sqrt{g_{1}(u)}-\frac{v}{u^{2}+v^{2}} \sqrt{g_{2}(v)} .
\end{array}
$$

where

$$
\begin{gathered}
g_{1}(u)=p_{u}^{2}=2\left(2-f+h u^{2}-\frac{\beta}{2} u^{4}-(\alpha+\gamma) u^{6}\right) \\
g_{2}(v)=p_{v}^{2}=2\left(f+h v^{2}+\frac{\beta}{2} v^{4}-(\alpha+\gamma) v^{6}\right)
\end{gathered}
$$

in these conditions, the equations of motion are

$$
\begin{aligned}
& \dot{u}=p_{u}= \pm \sqrt{g_{1}(u)}, \\
& \dot{v}=p_{v}= \pm \sqrt{g_{2}(v)} .
\end{aligned}
$$

$H=h$ and $F=f$ are the first integrals of motion, functions of $\left(u, v, p_{u}, p_{v}\right)$ which are constant along the solutions of Equation (11). The system is called (meromorphically) Liouville integrable or completely integrable.

\subsection{The Bifurcation Diagrams and the Admissible Region}

In this section we shall give the admissible region on the bifurcation diagrams 
and a detailed description of the real phase space topology i.e. the topology of the real level sets for all generic constants $h$ and $f$.

$$
A_{\mathbb{R}}=\left\{\left(u, v, p_{u}, p_{v}\right) \in \mathcal{R}^{4}: H=h, F=f\right\} \subset \mathcal{R}^{4}
$$

For doing that we find first the bifurcation diagram $B$ of the problem (12), i.e. the set of critical values of the energy-momentum mapping

$$
\left(u, v, p_{u}, p_{v}\right) \rightarrow(H, F)
$$

It appears, according to several works carried out in this sense (for details, see Ref. [22]-[27]), that the bifurcation diagram $B$ is exactly the discriminant locus of the polynomials $g_{1}(u)$ and $g_{2}(v)$ whose coefficients are functions in $h, f$ and the parameters of the system $(\alpha, \beta, \gamma)$.

$$
\begin{gathered}
B=B_{1} \cup B_{2} \\
B=\left\{(h, f, \alpha, \beta, \gamma) \in \mathcal{R}^{5}: \operatorname{discr}\left(g_{1}(u)\right)=0\right\} \\
\bigcup\left\{(h, f, \alpha, \beta, \gamma) \in \mathcal{R}^{5}: \operatorname{discr}\left(g_{2}(v)\right)=0\right\}
\end{gathered}
$$

It is convenient to consider separately, for some values of the constants $\alpha, \beta$ and $\gamma$, two cases $|\lambda| \geq 2$ and $|\lambda|<2$, because they generate different structures of the topology of the real level sets $A_{\mathbb{R}}$ and the corresponding Poincaré map.

3.1.1. For the Case $|\lambda| \geq 2$

$$
\begin{aligned}
& B_{1}=\left\{(h, f) \in \mathcal{R}^{2}: f=2, f=\frac{1348}{675}-\frac{h}{15} \pm \frac{2}{675} \sqrt{1+45 h+675 h^{2}+3375 h^{3}}\right\} \subset \mathcal{R}^{2} \\
& B_{2}=\left\{(h, f) \in \mathcal{R}^{2}: f=0, f=\frac{-2}{675}-\frac{h}{15} \pm \frac{2}{675} \sqrt{1+45 h+675 h^{2}+3375 h^{3}}\right\} \subset \mathcal{R}^{2}
\end{aligned}
$$

The set $\left\{\mathbb{R}^{3} \backslash B\right\} \cap\{|\lambda| \geq 2\}$ consists of 10 connected components (as it is shown in Figure 1). Thus, in each connected component of the set $A_{\mathbb{R}} \backslash B$ the level set $A_{\mathbb{R}}$ has the same topological type, and this latter may be changed only if $(h, f)$ passes through $B \cap\{|\lambda| \geq 2\}$.

Theorem 1. The set $\left\{\mathbb{R}^{3} \backslash B\right\} \cap\{|\lambda| \geq 2\}$ consists of ten connected components. The sections of these components with the plane $\{\lambda=$ const. $\}$ are shown on Figure 1. If $(h, f, \lambda) \in \mathbb{R}^{3} \backslash B$ the topological type of $A_{\mathbb{R}}$ is (diffeomorphic to) a two-dimensional tori $T$, to a disjoint union of two-dimensional two-tori $2 T$, or it is the empty set $\phi$ as it is shown in Table 1.

Proof. Consider the complexified system

$$
A_{\not \subset}=\left\{\left(x, z, p_{x}, p_{z}, r\right) \in \not \check{C}^{5}: H=h=\text { const. }, F=f=\text { const. }, r^{2}=x^{2}+z^{2}, r \neq 0\right\}
$$

consider also the hyperelliptic curves $\Gamma_{1}:\left\{\omega_{1}^{2}=g_{1}(u)\right\}$ and $\Gamma_{2}:\left\{\omega_{2}^{2}=g_{2}(v)\right\}$ and the corresponding Riemann surfaces $R_{1}$ and $R_{2}$ of the same genus $j_{1}=j_{2}=2$. We obtain the explicit solutions of the initial problem (11) by solving the Jacobi inversion problem [28]. 
Table 1. The Topological type of $A_{\mathbb{R}}$, and the admissible set for $(h, f) \in \mathbb{R}^{2} \backslash B$.

\begin{tabular}{ccccc}
\hline Domain & Roots of $g_{1}(u)$ & Roots of $g_{2}(v)$ & Accessible region & $A_{\mathbb{R}}$ \\
\hline $\mathbf{1}$ & 0 & $v_{1}<0<v_{2}$ & $\varnothing \times\left[v_{1}, v_{2}\right]$ & $\emptyset$ \\
$\mathbf{2}$ & $u_{1}<u_{2}<0<u_{3}<u_{4}$ & $v_{1}<0<v_{2}$ & {$\left[u_{1}, u_{2}\right] \cup\left[u_{3}, u_{4}\right] \times\left[v_{1}, v_{2}\right]$} & $2 T$ \\
$\mathbf{3}$ & $u_{1}<u_{2}<0<u_{3}<u_{4}$ & $v_{1}<0<v_{2}$ & {$\left[u_{1}, u_{2}\right] \cup\left[u_{3}, u_{4}\right] \times\left[v_{1}, v_{2}\right]$} & $2 T$ \\
$\mathbf{4}$ & $u_{1}<0<u_{2}$ & $v_{1}<0<v_{2}$ & {$\left[u_{1}, u_{2}\right] \times\left[v_{1}, v_{2}\right]$} & $T$ \\
$\mathbf{5}$ & $u_{1}<0<u_{2}$ & $v_{1}<0<v_{2}$ & {$\left[u_{1}, u_{2}\right] \times\left[v_{1}, v_{2}\right]$} & $T$ \\
$\mathbf{6}$ & $u_{1}<0<u_{2}$ & $v_{1}<0<v_{2}$ & {$\left[u_{1}, u_{2}\right] \times\left[v_{1}, v_{2}\right]$} & $T$ \\
$\mathbf{7}$ & $u_{1}<0<u_{2}$ & $v_{1}<0<v_{2}$ & {$\left[u_{1}, u_{2}\right] \times\left[v_{1}, v_{2}\right]$} & $T$ \\
$\mathbf{8}$ & $u_{1}<0<u_{2}$ & $v_{1}<v_{2}<0<v_{3}<v_{4}$ & {$\left[u_{1}, u_{2}\right] \times\left[v_{1}, v_{2}\right] \cup\left[v_{3}, v_{4}\right]$} & $2 T$ \\
$\mathbf{9}$ & $u_{1}<0<u_{2}$ & $v_{1}<v_{2}<0<v_{3}<v_{4}$ & {$\left[u_{1}, u_{2}\right] \times\left[v_{1}, v_{2}\right] \cup\left[v_{3}, v_{4}\right]$} & $2 T$ \\
$\mathbf{1 0}$ & $u_{1}<0<u_{2}$ & 0 & {$\left[u_{1}, u_{2}\right] \times \varnothing$} & $\emptyset$ \\
\hline
\end{tabular}

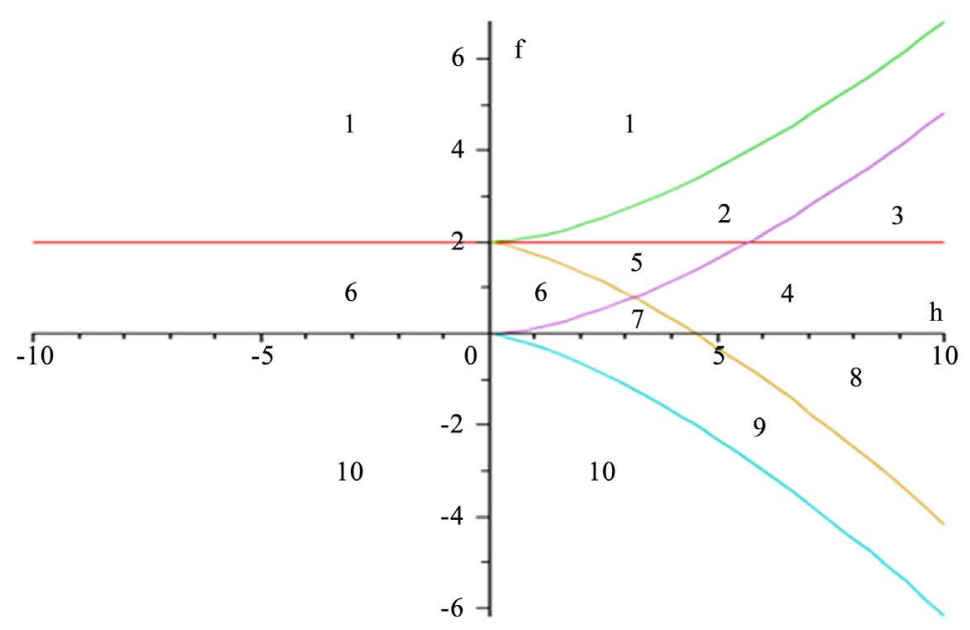

Figure 1. Bifurcation diagram $B \cap\{\lambda=$ const. $\}$ and the numbering of domains for $|\lambda| \geq 2$.

Thus $x, z, p_{x}, p_{z}$ can be expressed in terms of hyperelliptic functions living in the Jacobi variety $\Gamma=\Gamma_{1} \otimes \Gamma_{2}$ (where $\otimes$ is the symmetric product). These functions however are not single valued as can be seen from formulae (8) and (11).

Indeed, to each point on the symmetric product $\Gamma_{1} \otimes \Gamma_{2}$ there correspond two values of $\left(x, z, p_{x}, p_{z}\right)$. Thus we define the natural projection

$$
\pi: A_{\not} \rightarrow \Gamma_{1} \otimes \Gamma_{2}
$$

corresponding to the involution $i$

$$
i:\left(x, z, p_{x}, p_{z}\right) \rightarrow\left(x, z,-p_{x},-p_{z}\right)
$$

the real level sets $A_{\mathbb{R}}=\mathcal{R} e\left(A_{\varnothing}\right)$ is the set of fixed points of the complex conju- 
gation on $A_{\sqsubset}$ :

$$
\eta:\left(x, z, p_{x}, p_{z}\right) \rightarrow\left(\bar{x}, \bar{z}, \bar{p}_{x}, \bar{p}_{z}\right)
$$

Consider also the natural projection $\xi$ on the Riemann surface $R=R_{1} \otimes R_{2}$ given in $u, v$ coordinates by:

$$
\xi:(u, v) \rightarrow(\bar{u}, \bar{v})
$$

It induces an involution on the Jacobi variety and hence on $A_{\varangle}$ by the natural projection $\pi$. Formulae (6) and (7) imply that this involution $\xi$ coincides with the complex conjugation (15) on $A_{\subset}$ the upshot is that in order to describe $A_{\mathbb{R}}$ it is enough to study the projection:

$$
\pi: A_{\varnothing} \rightarrow J a c(R)=\Gamma_{1} \otimes \Gamma_{2}
$$

Definition. A connected component of the set of fixed points of $\eta$ on the curves $\Gamma_{1}$ and $\Gamma_{2}$ is called an oval.

To determine the ovals of $\Gamma_{1}$ and $\Gamma_{2}$ it suffices to study the real roots of the polynomials $g_{1}(u)$ and $g_{2}(v)$ for different values of $h$ and $f$ as shown in Table 1. Using the formulae (8) and the condition that $\left(x, z, p_{x}, p_{z}\right) \in \mathbb{R}^{4}$, we find exactly two admissible ovals whose projections on the $u$-plane and the $v$-plane are given by $\Delta_{1}$ and $\Delta_{2}$. The product of the admissible ovals in $\Gamma_{1} \otimes \Gamma_{2}$ and the projection $\pi$ of $A_{\mathbb{R}}$ such as, $A_{\mathbb{R}}=\pi^{-1}\left(\Gamma_{1} \otimes \Gamma_{2}\right)=\Delta_{1} \times \Delta_{2}$, gives:

3.1.2. For the Case $|\lambda|<2$

$$
\begin{aligned}
& B_{1}^{\prime}=\left\{(h, f) \in \mathcal{R}^{2}: f=2, f=\frac{484}{243}+\frac{h}{9} \pm \frac{2}{243} \sqrt{1-27 h+243 h^{2}-729 h^{3}}\right\} \subset \mathcal{R}^{2} \\
& B_{2}^{\prime}=\left\{(h, f) \in \mathcal{R}^{2}: f=0, f=\frac{-2}{243}+\frac{h}{9} \pm \frac{2}{243} \sqrt{1-27 h+243 h^{2}-729 h^{3}}\right\} \subset \mathcal{R}^{2}
\end{aligned}
$$

Theorem 2. The set $\left\{\mathbb{R}^{3} \backslash B^{\prime}\right\} \cap\{|\lambda|<2\}$ consists of ten connected components. The sections of these components with the plane $\{\lambda=$ const. $\}$ are shown on Figure 2. If $(h, f, \lambda) \in \mathbb{R}^{3} \backslash B^{\prime}$ The topological type of $A_{\mathbb{R}}$ is (diffeomorphic to) a two-dimensional tori $T$, to a disjoint union of cylinders $C$, or it is the real planes $\mathcal{R}^{2}$ as it is shown in Table 2.

\subsection{Topology of Singular Level Sets and Bifurcations of Liouville Tori}

Suppose now that the constants $h, f$ are changed in such a way that $(h, f)$ passes through the bifurcation diagram $B$. Then the topological type of $A_{\mathbb{R}}$ may change and the bifurcation of Liouville tori takes place.

For the first case $|\lambda| \geq 2$, According to Fomenko surgery on Liouville tori [29], we can have in our case three types of bifurcation of the level set $A_{\mathbb{R}}$ (see Table 3). To prove that, it suffices to look at the bifurcations of roots of the polynomials $g_{1}(u)$ and $g_{2}(v)$. The correspondence between bifurcation of roots and Liouville tori is shown in Figure 3. 


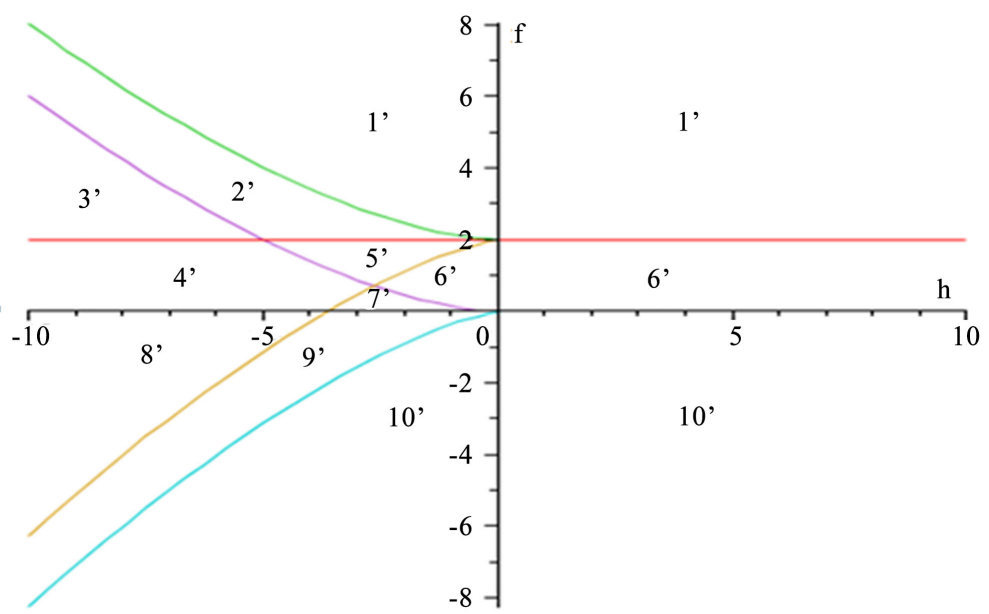

Figure 2. Bifurcation diagram $B^{\prime} \cap\{\lambda=$ const. $\}$ and the numbering of domains for $|\lambda|<2$.

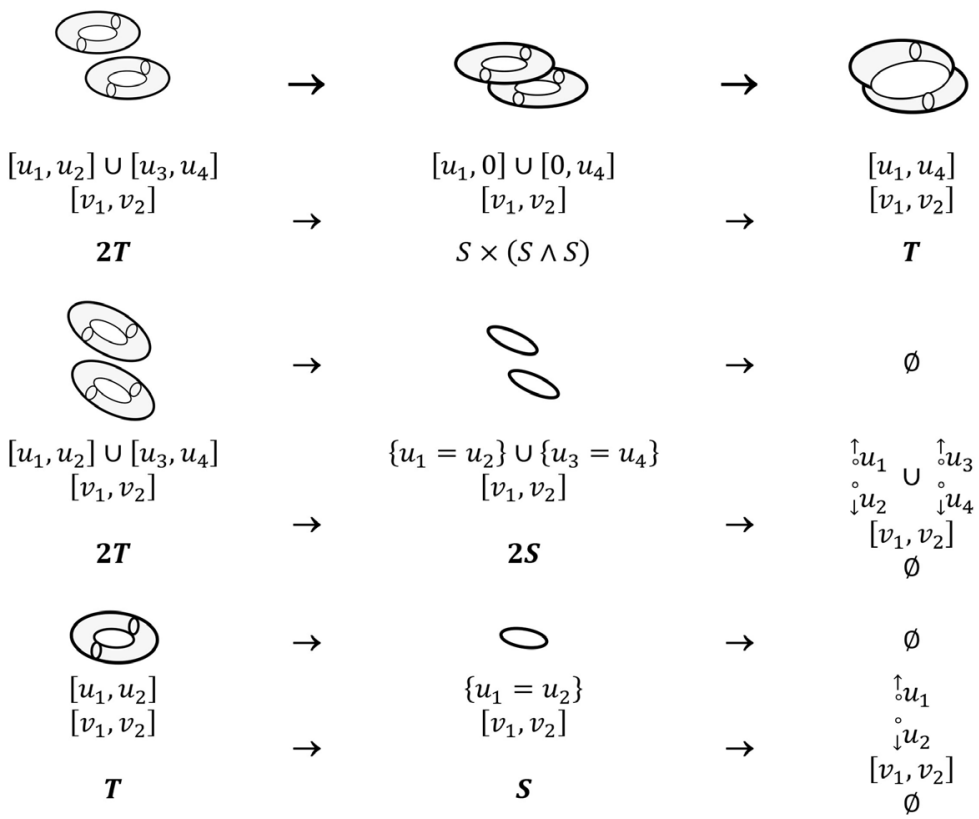

Figure 3. Correspondence between bifurcation sets for $(h, f) \in B$ and bifurcation of invariant Liouville tori, where $(S \wedge S)$ is a union of two circles having exactly one common point.

Table 2. The topological type of $A_{\mathbb{R}}$, and the admissible set for $(h, f) \in \mathbb{R}^{2} \backslash B^{\prime}$.

\begin{tabular}{ccc}
\hline Domain & Accessible region & $A_{\mathbb{R}}$ \\
\hline $1^{\prime}$ & ]$\left.-\infty, u_{1}\right] \cup\left[u_{2},+\infty[\times]-\infty,+\infty[\right.$ & $2 \mathcal{R}^{2}$ \\
$2^{\prime}$ & ]$\left.-\infty, u_{1}\right] \cup\left[u_{2},+\infty[\times]-\infty,+\infty[\right.$ & $2 \mathcal{R}^{2}$ \\
$3^{\prime}$ & ]$\left.-\infty, u_{1}\right] \cup\left[u_{2},+\infty[\times]-\infty, v_{1}\right] \cup\left[v_{2}, v_{3}\right] \cup\left[v_{4},+\infty[\right.$ & $2 C+4 \mathcal{R}^{2}$ \\
$4^{\prime}$ & ]$\left.-\infty, u_{1}\right] \cup\left[u_{2}, u_{3}\right] \cup\left[u_{4},+\infty[\times]-\infty, v_{1}\right] \cup\left[v_{2}, v_{3}\right] \cup\left[v_{4},+\infty[\right.$ & $T+4 C+4 \mathcal{R}^{2}$
\end{tabular}


Continued

\begin{tabular}{ccc}
\hline $5^{\prime}$ & ]$\left.-\infty, u_{1}\right] \cup\left[u_{2}, u_{3}\right] \cup\left[u_{4},+\infty[\times]-\infty,+\infty[\right.$ & $C+2 \mathcal{R}^{2}$ \\
$6^{\prime}$ & ]$-\infty,+\infty[\times]-\infty,+\infty[$ & $\mathcal{R}^{2}$ \\
7 & ]$\left.-\infty,+\infty[\times]-\infty, v_{1}\right] \cup\left[v_{2}, v_{3}\right] \cup\left[v_{4},+\infty[\right.$ & $C+2 \mathcal{R}^{2}$ \\
$8^{\prime}$ & ]$\left.-\infty, u_{1}\right] \cup\left[u_{2}, u_{3}\right] \cup\left[u_{4},+\infty[\times]-\infty, v_{1}\right] \cup\left[v_{2},+\infty[\right.$ & $2 C+4 \mathcal{R}^{2}$ \\
9 & ]$\left.-\infty,+\infty[\times]-\infty, v_{1}\right] \cup\left[v_{2},+\infty[\right.$ & $2 \mathcal{R}^{2}$ \\
$10^{\prime}$ & ]$\left.-\infty,+\infty[\times]-\infty, v_{1}\right] \cup\left[v_{2},+\infty[\right.$ & $2 \mathcal{R}^{2}$ \\
\hline
\end{tabular}

Table 3. Generic bifurcations of the level set $A_{\mathbb{R}}$.

\begin{tabular}{ccc}
\hline $6 \rightarrow 1$ & $2 \rightarrow 1$ & $2 \rightarrow 5,9 \rightarrow 10$ \\
$6 \rightarrow 10$ & $9 \rightarrow 10$ & $3 \rightarrow 4,8 \rightarrow 4$ \\
\hline$T \rightarrow \phi$ & $2 T \rightarrow \phi$ & $2 T \rightarrow T$ \\
\hline
\end{tabular}

As shown in Figure 3, three types of bifurcations of (surgery on) Liouville tori take place:

1) Bifurcation $T \rightarrow S \rightarrow \varnothing:$ The two dimensional tori $T$ is contracted to the circle $S$ corresponding to the periodic solution, and then vanishes.

2) Bifurcation $2 T \rightarrow S \times(S \wedge S) \rightarrow T$ : The two dimensional two tori $2 T$ merge into two dimensional tori $T$ by passing through the complex $S \times(S \wedge S)$ where $(S \wedge S)$ is a union of two circles having exactly one common point.

3) Bifurcation $2 T \rightarrow 2 S \rightarrow \varnothing$ : The two dimensional two tori $2 T$ are contracted to two circles $2 S$ corresponding to two periodic solutions, and then vanishes.

For the second case $|\lambda|<2$, The Fomenko classification of bifurcation of Liouville tori [29] cannot be applied as its invariant level sets contain a non-compact component (cylinder $C$ or plane $\mathcal{R}^{2}$ ). Thus, we can have the type of bifurcation of the level set $A_{\mathbb{R}}$ passing from domain $i$ to domain $j$ in Table 4 .

To prove that, it suffices to look at the bifurcation of roots of the polynomials $g_{1}(u)$ and $g_{2}(v)$ as shown in Figure 4.

\section{Periodic Solutions}

\subsection{For the Case $|\lambda| \geq 2$}

When the bifurcation of Liouville tori takes place, the level set $A_{\mathbb{R}}$ becomes completely degenerate. Then we can have exceptional families of periodic solutions. It is seen from Table 5 , that if $(h, f)$ is on the smooth curves $C_{0}\left(C_{0}^{\prime}\right)$ or $C_{1}\left(C_{1}^{\prime}\right)$ (see Figure 5 ), $A_{\mathbb{R}}$ contains a isolated circles $S$ and $2 S$, respectively which are periodic solutions. Consider now a fixed periodic solution belonging to the curve $C_{0}$. The parameter $v$ takes values in the admissible interval $\left[v_{1}, v_{2}\right]$ and $u=u_{1}=u_{2}$ is equal to the double root of the polynomial $g_{1}(u)$ (see Table 5). 
Then we obtain from Equation (8) the following parameterization of fixed periodic solution:

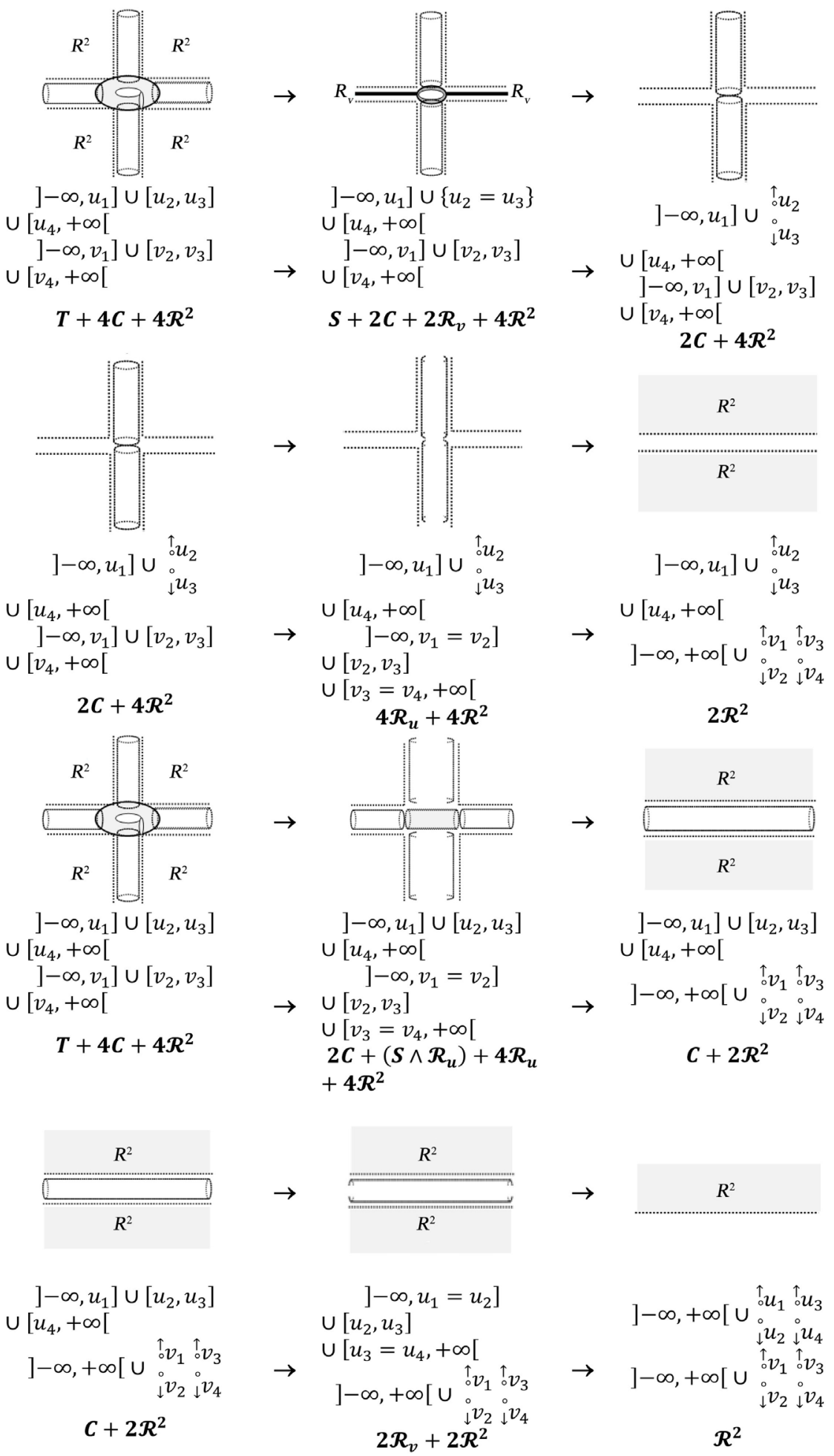

Figure 4. Correspondence between bifurcation of $A_{\mathbb{R}}$ and bifurcation of roots of the polynomials $g_{1}(u)$ and $g_{2}(v)$ for differnt values of the invariants $(h, f)$ on the bifurcation diagram $B^{\prime}$. 


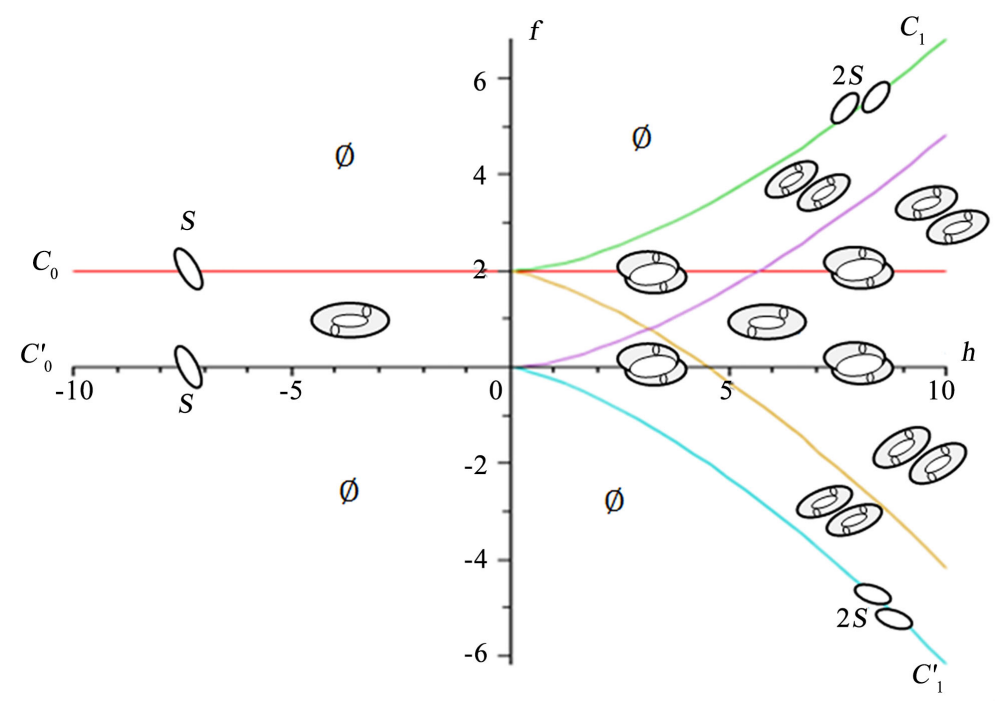

Figure 5. Topological type of $A_{\mathbb{R}}$ for $(h, f) \in B$ and $|\lambda| \geq 2$.

Table 4. Generic bifurcations of the level set $A_{\mathbb{R}}$.

\begin{tabular}{cccc}
\hline $4^{\prime} \rightarrow 3^{\prime}$ & $4^{\prime} \rightarrow 5^{\prime}$ & $3^{\prime} \rightarrow 2^{\prime}$ & $5^{\prime} \rightarrow 6^{\prime}$ \\
$4^{\prime} \rightarrow 8^{\prime}$ & $4^{\prime} \rightarrow 7^{\prime}$ & $8^{\prime} \rightarrow 9^{\prime}$ & $7^{\prime} \rightarrow 6^{\prime}$ \\
\hline$T+4 C+4 \mathcal{R}^{2} \rightarrow 2 C+4 \mathcal{R}^{2}$ & $T+4 C+4 \mathcal{R}^{2} \rightarrow C+2 \mathcal{R}^{2}$ & $2 C+4 \mathcal{R}^{2} \rightarrow 2 \mathcal{R}^{2}$ & $C+2 \mathcal{R}^{2} \rightarrow 2 \mathcal{R}^{2}$
\end{tabular}

Table 5. Topological type of $A_{\mathbb{R}}$ for $(h, f) \in B$.

\begin{tabular}{rcc}
\hline Curves & Accessible region & $A_{\mathbb{R}}$ \\
\hline$C_{0}$ & $\left\{u_{1}=u_{2}\right\} \times\left[v_{1}, v_{2}\right]$ & $S$ \\
$C_{1}$ & $\left\{u_{1}=u_{2}\right\} \cup\left\{u_{3}=u_{4}\right\} \times\left[v_{1}, v_{2}\right]$ & $2 S$ \\
$C_{0}^{\prime}$ & {$\left[u_{1}, u_{2}\right] \times\left\{v_{1}=v_{2}\right\}$} & $S$ \\
$C_{1}^{\prime}$ & {$\left[u_{1}, u_{2}\right] \times\left\{v_{1}=v_{2}\right\} \bigcup\left\{v_{3}=v_{4}\right\}$} & $2 S$ \\
\hline
\end{tabular}

$$
\left\{\begin{array} { l } 
{ x = 0 } \\
{ z = \frac { - v ^ { 2 } } { 2 } }
\end{array} \text { and } \left\{\begin{array}{l}
p_{x}=0 \\
p_{z}= \pm \frac{\sqrt{g_{2}(v)}}{v}
\end{array}\right.\right.
$$

to derive the differential equation satisfied by $v$, we use

$$
\frac{\mathrm{d} v}{\mathrm{~d} \tau}=p_{v}
$$

we obtain from (10)

$$
\frac{\mathrm{d} v}{\sqrt{g_{2}(v)}}= \pm \mathrm{d} \tau
$$

thus $v(\tau)$ and hence $z(\tau)$ can be expressed in terms of hyperelliptic functions. 
On the curve $C_{0}$, the second integral of motion $f$ is equal to 2 , as well as the characteristic polynomial $g_{2}(v)=p_{v}^{2}=2\left(2+h v^{2}+v^{4}-5 v^{6}\right)$ depends only on $h$. Taking this variable change:

$$
\begin{gathered}
R=v^{2} \Rightarrow \frac{\mathrm{d} R}{2 \sqrt{R}}=\mathrm{d} v \\
\mathrm{~d} \tau= \pm \frac{\mathrm{d} R}{2 \sqrt{2} \sqrt{R\left(2+h R-5 R^{3}+R^{2}\right)}}= \pm \frac{\mathrm{d} R}{2 \sqrt{2} \sqrt{P_{2}(R)}}
\end{gathered}
$$

the polynomial $P_{2}(R)$ has four distinct roots

$$
R_{0}=0, \quad R_{1}=\frac{D^{\frac{1}{3}}}{30}+\frac{2\left(h+\frac{1}{15}\right)}{D^{\frac{1}{3}}}+\frac{1}{15}, R_{2}=a_{0}+i b_{0} \text { et } R_{3}=a_{0}-i b_{0}
$$

where

$$
\begin{gathered}
a_{0}=\frac{-D^{\frac{1}{3}}}{60}-\frac{\left(h+\frac{1}{15}\right)}{D^{\frac{1}{3}}}+\frac{1}{15}, b_{0}=\frac{\sqrt{3}}{2}\left[\frac{-D^{\frac{1}{3}}}{60}-\frac{\left(h+\frac{1}{15}\right)}{D^{\frac{1}{3}}}\right] \\
D=180 h+5408+60 \sqrt{-60 h^{3}-3 h^{2}+540 h+8124}
\end{gathered}
$$

By an inversion of the elliptic integral Equation (17), one explicitly obtains the expression the periodic solution $v(\tau)$ :

$$
\begin{gathered}
\oint_{0}^{\tau} \mathrm{d} \tau=\frac{1}{2 \sqrt{2}} \int_{R_{0}}^{R} \frac{\mathrm{d} R}{\sqrt{\left(R-R_{0}\right)\left(R-R_{1}\right)\left(R-R_{2}\right)\left(R-R_{3}\right)}} \\
\tau=n_{0} C n^{-1}\left(\cos \varphi, k_{0}\right)
\end{gathered}
$$

where

$$
n_{0}=\frac{1}{2 \sqrt{2 A_{0} B_{0}}}, k_{0}=\frac{R_{1}^{2}-\left(A_{0}-B_{0}\right)^{2}}{4 A_{0} B_{0}}, A_{0}^{2}=R_{1}^{2}+a_{0}^{2}, B_{0}^{2}=b_{0}^{2}+a_{0}^{2}
$$

$C n^{-1}\left(\cos \varphi, k_{0}\right)=F\left(\varphi, k_{0}\right)$ being the incomplete elliptic integral of first kind. The expression of periodic solution $v(\tau)$ is given by solving the Jacobi inversion problem:

$$
\tau=\int^{\tau} \mathrm{d} t=\int \frac{\mathrm{d} v}{\sqrt{g_{2}(v)}} \Rightarrow v(\tau)= \pm \sqrt{\frac{R_{1} B_{0}\left\{C n\left(2 t \sqrt{2 A_{0} B_{0}}\right)-1\right\}}{\left(A_{0}+B_{0}\right)+\left(A_{0}-B_{0}\right) C n\left(2 t \sqrt{2 A_{0} B_{0}}\right)}}
$$

The period $T_{v}$ associated with the solution $v(t)$ is obtained by calculating the elliptic integral Equation (17) over the totality of the admissible oval for $v$.

$$
T_{v}=\oint \mathrm{d} \tau=\frac{1}{2 \sqrt{2}} \oint \frac{\mathrm{d} R}{\sqrt{P_{2}(R)}}=\frac{2}{2 \sqrt{2}} \int_{R_{0}}^{R_{1}} \frac{\mathrm{d} p_{y}}{\sqrt{R\left(2+h R-5 R^{3}+R^{2}\right)}}
$$

It's sufficient to replace in Equation (18) the upper bound in the integral by $R_{1}$ and multiply by 2 , in these conditions $\cos \varphi=-1$, we obtain a period de- 
pendent only on the energy $h$ :

$$
T_{v}(h)=2 n_{0} C n^{-1}\left(-1, k_{0}\right)=2 n_{0} F\left(\pi, k_{0}\right)
$$

where $C n^{-1}\left(-1, k_{0}\right)=F\left(\pi, k_{0}\right)$ is the complete elliptic integral of the first kind.

In the same way, for the periodic solution on the curve $C_{1}$ the parameter $V$ takes values in the admissible oval $\left[v_{1}, v_{2}\right]$, the double roots of the polynomial $g_{1}(u)$ are equal to $u_{1}=u_{2}=-m$ and $u_{3}=u_{4}=m$ (see Table 5).

The values of the first integrals $H$ and $F$ on the curve $C_{1}$ are related by

$$
f=\frac{1348}{675}-\frac{h}{15}+\frac{2}{675} \sqrt{1+45 h+675 h^{2}+3375 h^{3}}
$$

From Equation (8) we obtain the following parameterization of fixed periodic solution

$$
\begin{aligned}
& \left\{\begin{array} { l } 
{ x = \pm m v } \\
{ z = \frac { m ^ { 2 } - v ^ { 2 } } { 2 } }
\end{array} \text { and } \left\{\begin{array}{l}
p_{x}= \pm \frac{m}{m^{2}+v^{2}} \sqrt{g_{2}(v)} \\
p_{z}= \pm \frac{v}{m^{2}+v^{2}} \sqrt{g_{2}(v)}
\end{array}\right.\right. \\
& v(t)= \pm \sqrt{\frac{R_{1} B_{1}\left\{C n\left(2 t \sqrt{2 A_{1} B_{1}}\right)-1\right\}}{\left(A_{1}+B_{1}\right)+\left(A_{1}-B_{1}\right) C n\left(2 t \sqrt{2 A_{1} B_{1}}\right)}}
\end{aligned}
$$

The period of $v(t)$ is

$$
T_{v}(h)=2 n_{1} C n^{-1}\left(-1, k_{1}\right)=2 n_{1} F\left(\pi, k_{1}\right)
$$

where

$$
\begin{gathered}
n_{1}=\frac{1}{2 \sqrt{2 A_{1} B_{1}}}, k_{1}=\frac{R_{1}^{2}-\left(A_{1}-B_{1}\right)^{2}}{4 A_{1} B_{1}}, A_{1}^{2}=R_{1}^{2}+a_{1}^{2}, B_{1}^{2}=b_{1}^{2}+a_{1}^{2}, \\
R_{0}=0, R_{1}=\frac{1}{15}\left(C^{\frac{1}{3}}+\frac{15 h+1}{C^{\frac{1}{3}}}+1\right), a_{1}=\frac{-1}{30}\left(C^{\frac{1}{3}}+\frac{15 h+1}{C^{\frac{1}{3}}}-2\right), \\
b_{1}=\frac{\sqrt{3}}{30}\left(C^{\frac{1}{3}}-\frac{15 h+1}{C^{\frac{1}{3}}}\right), \\
+\sqrt{911249+20503080 h+307546200 h^{2}+1537731000 h^{3}+911250 \sqrt{(15 h+1)^{3}}}
\end{gathered}
$$

From Equation (19), the Cartesian equation for this periodic solution in the $(x, z)$ plan is

$$
z(x)=\frac{m^{2}}{2}-\frac{x^{2}}{2 m^{2}}
$$

\subsection{For the Case $|\lambda|<2$}

In this case, it is seen from Table 6, that on the smooth curves $L_{0}\left(L_{0}^{\prime}\right)$ the 
values of the second integral of motion $f$ is equal to 2 ( $f$ is equal to 2 ) (see Figure 6), $A_{\mathbb{R}}$ contains a circles $S$ which correspond to a periodic solutions. On the curve $L_{0}(f=2)$ the periodic solution $v(t)$ is given by

$$
v(t)= \pm \sqrt{\frac{R_{1} B_{0}^{\prime}\left\{C n\left(2 t \sqrt{2 A_{0}^{\prime} B_{0}^{\prime}}\right)-1\right\}}{\left(A_{0}^{\prime}+B_{0}^{\prime}\right)+\left(A_{0}^{\prime}-B_{0}^{\prime}\right) C n\left(2 t \sqrt{2 A_{0}^{\prime} B_{0}^{\prime}}\right)}}
$$

The associated period is

$$
T_{v}(h)=2 n_{0}^{\prime} C n^{-1}\left(-1, k_{0}^{\prime}\right)=2 n_{0}^{\prime} F\left(\pi, k_{0}^{\prime}\right)
$$

where

$$
\begin{gathered}
n_{0}^{\prime}=\frac{1}{2 \sqrt{2 A_{0}^{\prime} B_{0}^{\prime}}}, k_{0}^{\prime}=\frac{R_{1}^{2}-\left(A_{0}^{\prime}-B_{0}^{\prime}\right)^{2}}{4 A_{0}^{\prime} B_{0}^{\prime}}, A_{0}^{\prime 2}=R_{1}^{2}+a_{0}^{\prime 2}, B_{0}^{\prime 2}=b_{0}^{\prime 2}+a_{0}^{\prime 2}, R_{0}=0, \\
R_{1}=\frac{D_{0}^{\frac{1}{3}}}{18}-\frac{2\left(h-\frac{1}{9}\right)}{D_{0}^{\frac{1}{3}}}-\frac{1}{9}, a_{0}=\frac{-D_{0}^{\frac{1}{3}}}{36}+\frac{\left(h-\frac{1}{9}\right)}{D_{0}^{\frac{1}{3}}}-\frac{1}{9}, b_{0}=\frac{\sqrt{3}}{2}\left(\frac{D_{0}^{\frac{1}{3}}}{18}+\frac{2\left(h-\frac{1}{9}\right)}{\left.D_{0}^{\frac{1}{3}}\right)}\right) \\
D_{0}=108 h-1952+36 \sqrt{36 h^{3}-3 h^{2}-324 h+2940}
\end{gathered}
$$

\begin{tabular}{|c|c|c|}
\hline Curves & Accessible region & $A_{\mathbb{R}}$ \\
\hline$L_{0}$ & $\begin{array}{l}\left.]-\infty, u_{1}\right] \cup\left\{u_{2}=u_{3}\right\} \cup\left[u_{4},+\infty[\right. \\
\left.\times]-\infty, v_{1}\right] \cup\left[v_{2}, v_{3}\right] \cup\left[v_{4},+\infty[\right.\end{array}$ & $S+2 \mathcal{R}_{v}+2 C+4 \mathcal{R}^{2}$ \\
\hline$L_{0}^{\prime}$ & $\begin{array}{l}\left.]-\infty, u_{1}\right] \cup\left[u_{2}, u_{3}\right] \cup\left[u_{4},+\infty[\right. \\
\left.\times]-\infty, v_{1}\right] \cup\left\{v_{2}=v_{3}\right\} \cup\left[v_{4},+\infty[\right.\end{array}$ & $S+2 \mathcal{R}_{u}+2 C+4 \mathcal{R}^{2}$ \\
\hline
\end{tabular}

Table 6. Topological type of $A_{\mathbb{R}}$ for $(h, f) \in B^{\prime}$.

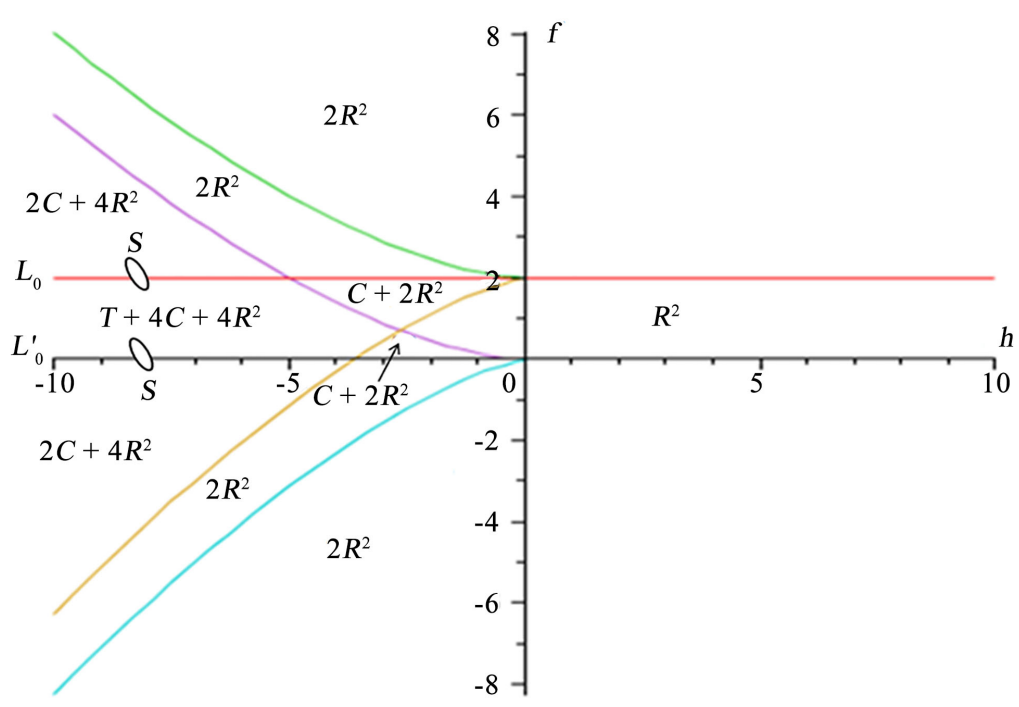

Figure 6. Topological type of $A_{\mathbb{R}}$ for $(h, f) \in B^{\prime}$ and $|\lambda|<2$. 


\section{Numerical Investigation}

By making use a set of software routines, implemented in Maple, for plotting 2D projections of Poincaré surfaces of section, method introduced by Poincaré and extended by Hénon [30], we give numerical illustrations of the real phase space topology studied in Section 3. For fixed values of constants $h, f, \alpha, \lambda$ and $\beta, \gamma$ vary, the Liouville tori contained in the level set $\{H=h, F=f\}$ change their topological type, while preserving the integrable behavior of the problem. However, with increasing control parameter associated with magnetic field, the random scattering of points in the sections shows that the system has carried out a transition from regularity through a quasi-regularity to chaotical dynamics.

The Poincaré surfaces of section are plotted in the plane $\left(q_{1}, p_{1}\right)=\left(u, p_{u}\right)$.

\subsection{Regularity-Chaos Transition}

Figures 7(a)-(h) represent Poincaré surfaces of section for $\beta=2$ (electric field) and values of $\gamma$ (magnetic field) near the integrable case $\gamma=4$ (from left to right), where for different values of $h$ and $f$ on the bifurcation diagram $B$ the system is totally regular, the topological type of $A_{\mathbb{R}}$ is diffeomorphic to a two-dimensional tori $T$, or a disjoint union of two-dimensional two-tori $2 T$. When the magnetic field increases for $\gamma=4$ to $\gamma=4.5$, the corresponding Poincaré surfaces of section show that the system go through a transition from regularity to chaotical dynamics. At $\gamma=5$ (high magnetic field), all Poincaré surfaces of section show a strong irregular motion, the dynamic is totally chaotic.

Figures 8(a)-(h) show the Poincaré surfaces of section for $\beta=0 \quad$ (no electric field) and $\gamma=4$, the system still has a regular motion, so we can say that the electric field is not responsible for the chaotic behavior of the system, on the other hand only the variation of the intensity of the magnetic field changes the behavior of the system from regularity to chaotically dynamics.

\subsection{Bifurcations of Liouville Tori}

Figure 9(a) represents the Poincaré surfaces of section for the bifurcation $2 T \rightarrow 2 S \rightarrow \phi$, when the system evolves from domain 2 to domain 1 on the bifurcation diagram where the values of the integrals of motion are $h=4.685$ and $f=2.221$ (blue), 2.524 (black), 2.793 (red), 3.363 (orange), 3.467 (black dot). Figure 9(b) represents the bifurcation $2 T \rightarrow 2 S \rightarrow \phi$ from domain 9 to domain 10 on the bifurcation diagram where $h=5.437$ and $f=-1.276$ (black), -1.847 (blue), -2.4 (red), -2.587 (black dot). The last values which correspond to black dots are located for $(h, f) \in B$, exactly on the curve of the bifurcation diagram, these black dots present periodic solutions in the form of two isolated circles, because the intersection of a circle with the cutting surface gives a dots. Figures 9 (c) represents the bifurcation $2 T \rightarrow S \times(S \wedge S) \rightarrow T$, from domain 3 to domain 4 where $h=8.873$ and $f=1.7$ (black), 2 (blue), 3.096 (red). Figures 9(d) represent the bifurcation $2 T \rightarrow S \times(S \wedge S) \rightarrow T$, from 

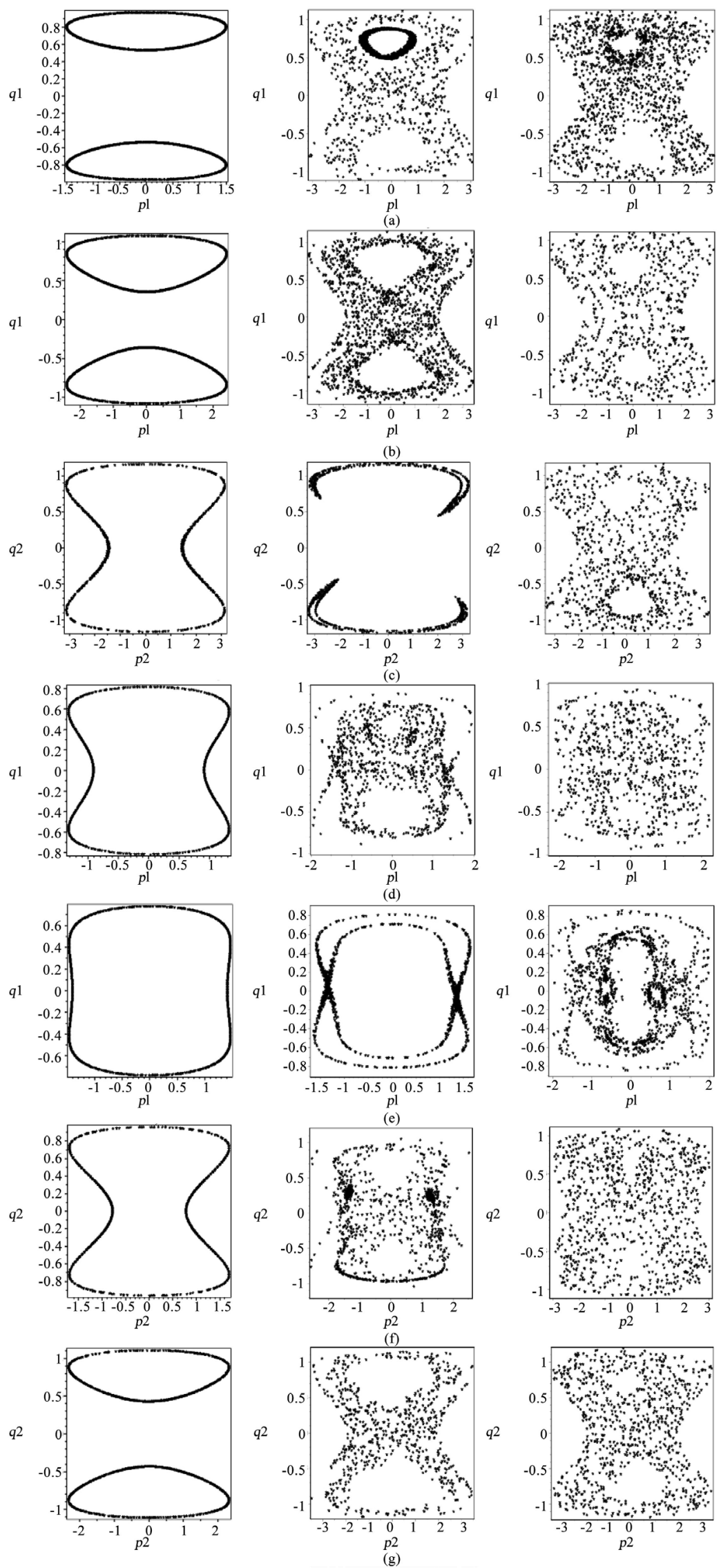

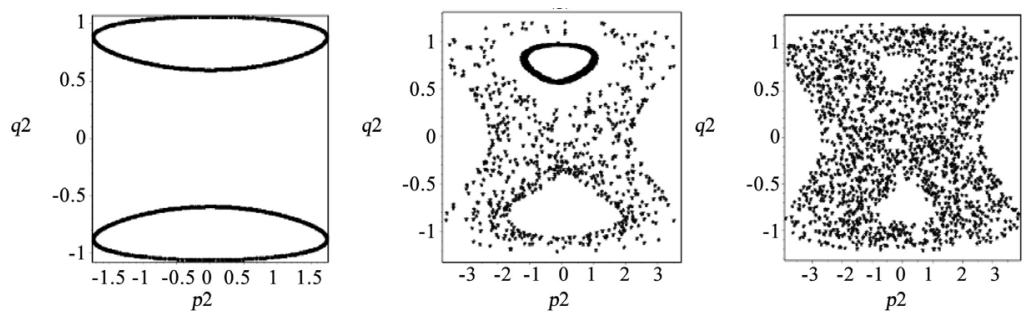

(h)

Figure 7. Poincaré surfaces of section for $(h, f) \in \mathbb{R}^{2} \backslash B$ and $|\lambda| \geq 2$. The three surfaces (from left to right) correspond to $\beta=2$ (electric field) and $\gamma=4,4.5,5$ (magnetic field). (a) Domain $2 \quad(h=7.477, f=3.936) A_{\mathbb{R}} \approx 2 T \quad$; (b) Domain 3 $(h=8.873, f=3.096) A_{\mathbb{R}} \approx 2 T$; (c) Domain $4(h=7.155, f=1.112) A_{\mathbb{R}} \approx T$; (d) Domain 5 $(h=2.269, f=1.583) A_{\mathbb{R}} \approx T$; (e) Domain $6(h=0.819, f=1.011) A_{\mathbb{R}} \approx T$; (f) Domain 7 $(h=3.021, f=0.305) A_{\mathbb{R}} \approx T ;(\mathrm{g})$ Domain $8(h=7.477, f=-1.376) A_{\mathbb{R}} \approx 2 T ;(\mathrm{h})$ Domain $9(h=7.477, f=-2.553) A_{\mathbb{R}} \approx 2 T$.
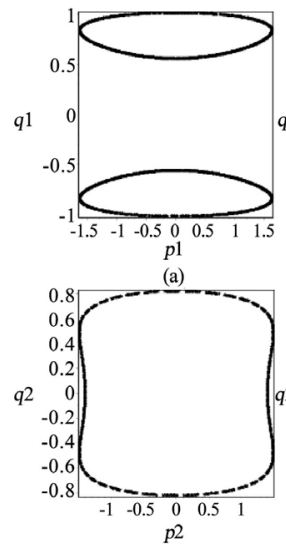

(a)
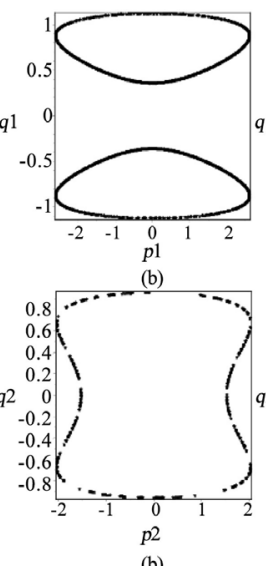
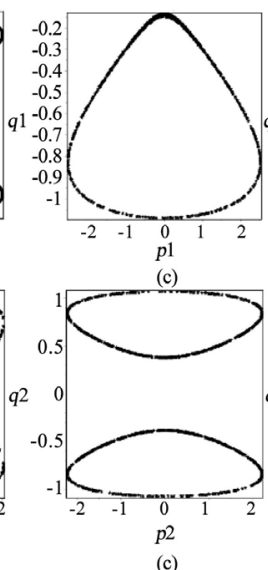
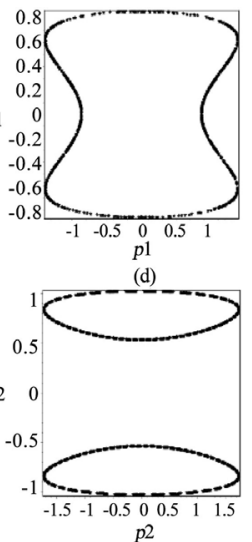

(d)

Figure 8. Poincaré surfaces of section for $(h, f) \in \mathbb{R}^{2} \backslash B$ and $|\lambda| \geq 2$. The surfaces correspond to $\beta=0$ (no electric field) and $\gamma=4$ (magnetic field). (a) Domain 2; (b) Domain 3; (c) Domain 4; (d) Domain 5; (e) Domain 6; (f) Domain 7; (g) Domain 8; (h) Domain 9.

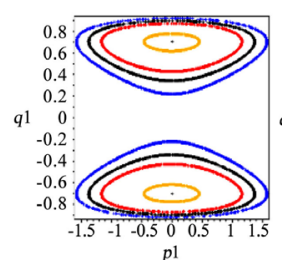

p1

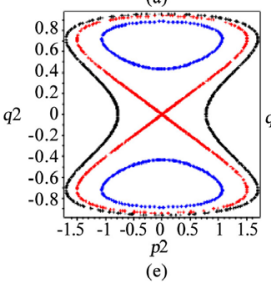

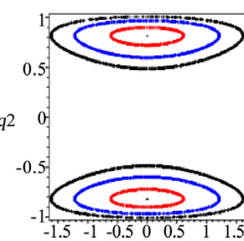

p2

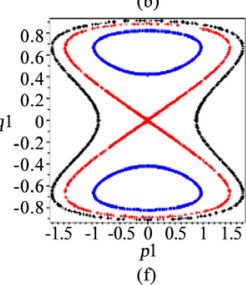

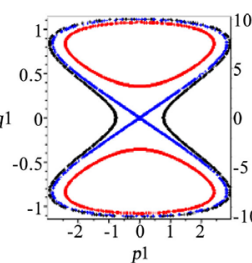

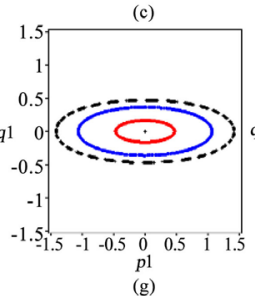

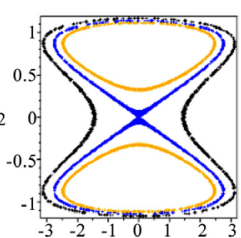

2

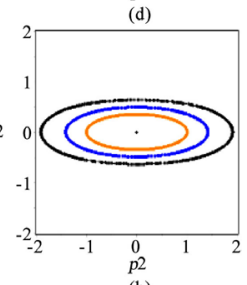

(h)

Figure 9. Poincaré surfaces of section on the bifurcation diagram for $(h, f) \in B$ and $|\lambda| \geq 2$. 
domain 8 to domain 4 where $h=7.155$ and $f=-0.738$ (black), 0 (blue), 1.112 (orange). Figures 9 (e) represents the bifurcation $2 T \rightarrow S \times(S \wedge S) \rightarrow T$, from domain 9 to domain 7 where $h=3.021$ and $f=-0.569$ (black), 0 (red), 0.305 (blue). Figures 9(f) represents the bifurcation $2 T \rightarrow S \times(S \wedge S) \rightarrow T$, from domain 2 to domain 5 where $h=3.88$ and $f=1.616$ (black), 2 (red), 2.625 (blue). Figures 9 (g) represents the bifurcation $T \rightarrow S \rightarrow \phi$, from domain 6 to domain 1 where $h=-4.067$ and $f=1$ (black), 1.437 (blue), 1.891 (red), 2 (black dot). Figures $9(\mathrm{~h})$ represents the bifurcation $T \rightarrow S \rightarrow \phi$, from domain 6 to domain 10 where the values of integrals of motion are $h=-4.067$ and $f=1$ (black), 0.5 (blue), 0.2 (red), 0 (black dot). The black dots correspond to the periodic solutions in the form of a isolated circles.

For the case $|\lambda|<2$, the invariant level sets contain a non-compact component (cylinder $C$ or plane $\mathcal{R}^{2}$ ), only in the domain 4 ' on the bifurcation diagram $B^{\prime}$ we find a single tori.

Figure 10 (a) shows the Poincaré sections for $\lambda= \pm \sqrt{3}$ and for a values of the integrals of motion $h=-7.879$ and $f=0.825$ (black), 1.122 (red), 1.758 (blue) corresponding to a point of domain 4' on the bifurcation diagram $B$, where $A_{\mathbb{R}}$ is a two-dimensional tori $T$.

Figure 10(b) shows the Poincaré sections for $\lambda= \pm \sqrt{3}$ corresponding to the bifurcation $T+4 C+4 \mathcal{R}^{2} \rightarrow S+2 \mathcal{R}_{u}+2 C+4 \mathcal{R}^{2} \rightarrow 2 C+4 \mathcal{R}^{2}$ from domain 4 ' to domain 3' on the bifurcation diagram $B$ ', where the values of the integrals of motion are $h=-7.879$ and $f=1.9$ (black), 2 (black dot), this black dot concerns the periodic solution in the form of a circle.

\section{Conclusion}

In this paper, we have studied the classical dynamics of the Hydrogen atom in the generalized van der Waals potential subjected to external parallel magnetic and electric fields. By making use of some reductions, the regularized Hamiltonian of the system is described by a two-degree of freedom dependent on certain control parameters. The converted system is equivalent to the motion of two coupled anharmonic oscillators with pseudo-energy easily exploitable. The
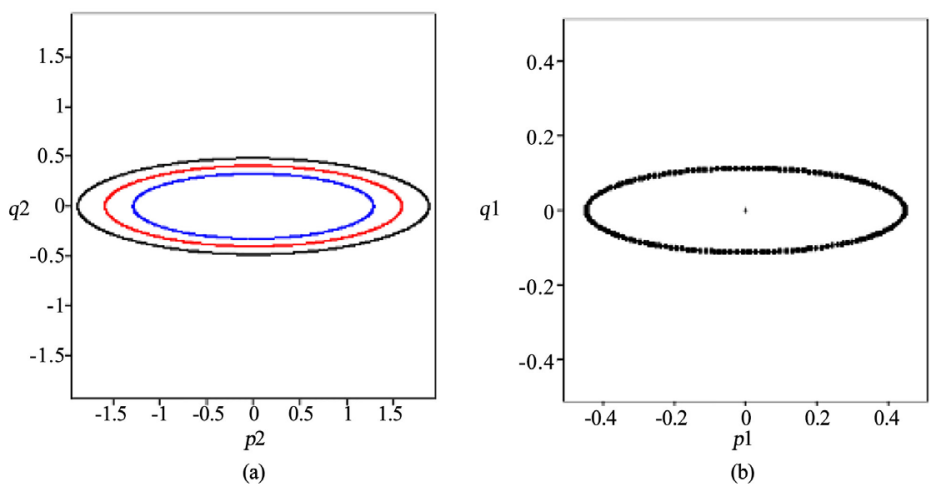

Figure 10. Poincaré surfaces of section for different values of, $h, f$ on the bifurcation diagram $B^{\prime}$ and $|\lambda|<2$. 
different results obtained show the capacity of the method used to provide precise information on this Hamiltonian system. The very important question that we have studied is the topological analysis of the real invariant manifolds $A_{\mathbb{R}}=\{H=h, F=f\}$ of the system. Fomenko's theory on surgery and bifurcations of the Liouville tori has been combined with that of the algebraic structure to give a rigorous and detailed description of the topology of invariant manifolds $A_{\mathbb{R}}$. For non-critical values of $H$ and $F$, and for some values of system parameters, we have distinguished two different cases $|\lambda| \geq 2$ where $A_{\mathbb{R}}$ contains torus or is empty. On the other hand, for the case $|\lambda|<2$, it consists of a torus, or a cylinder or a real plane. Indeed, for the first case, all the components of $A_{\mathbb{R}}$ are compact, while for the second case, they are not compact. Similarly, we have found that Fomenko's theory of the bifurcations of the Liouville tori is applicable to the first case, whereas for the second case, the bifurcations of the tori that appear cannot be described by this theory. It needs to be due to the fact that the Fomenko classification theorem on the bifurcations of the Liouville tori is only valid in the case where the invariant manifold $A_{\mathbb{R}}$ is formed only of compact components. We have also shown how the periodic orbits can be found, how the period of the solutions is determined, and in what ways explicit formulas can be established. Finally, we have also numerically illustrated the generic bifurcations of the Liouville tori and the regularity-chaos transition when one of the control parameters varies. The numerical results show that only the magnetic interaction has affected the dynamic behavior of the Hydrogen atom in parallel magnetic and electric fields. When the magnetic field is weak, the system is totally regular. When the magnetic field is increasing, we observe a transition from regularity to chaotical dynamics. For high magnetic field, the dynamic is totally chaotic.

\section{References}

[1] Popov, V.S. and Karnacov, B.M. (2014) Hydrogen Atom in a Strong Magnetic Field. Physics-Uspekhi, 57, 257-279. https://doi.org/10.3367/UFNe.0184.201403e.0273

[2] Stevanovic, L. and Melojevic, D. (2012) Compressed Hydrogen Atom under Debye Screening in Strong Magnetic Field. Publications of the Astronomical Observatory of Belgrade, 91, 327-330.

[3] Li, B. and Liu, H.P. (2013) An Effective Quantum Defect Theory for the Diamagnetic Spectrum of a Barium Rydberg Atom. Chinese Physics B, 22, 1. https://doi.org/10.1088/1674-1056/22/1/013203

[4] Shabab, A.E. and Usov, V.V. (2007) Modified Coulomb Law in a Strongly Magnetized Vacuum. Physical Review Letters, 98, Article ID: 180403.

[5] Wang, D. (2010) Dynamics of a Rydberg Hydrogen Atom in a Generalized van der Waals Potential and a Magnetic Field. Chinese Physics Letters, 27, Article ID: 023201.

[6] Iñarrea, M., Barrasa, V.L., Palacian, J. and Yanguas, P. (2007) Rydberg Hydrogen Atom near a Metallic Surface: Stark Regime and Ionization Dynamics. Physical Re view $A$, 76, Article ID: 052903.

[7] Iñarrea, M. and Salas, J.P. and Barrasa, V.L. (2002) Hydrogen Atom in the Presence of Uniform Magnetic and Quadrupolar Electric Fields: Integrability, Bifurcations, 
and Chaotic Behavior. Physical Review E, 66, Article ID: 056614.

[8] Salas, J.P. and Simonovic, N.S. (2000) Rydberg States of the Hydrogen Atom in the Instantaneous van der Waals Potential: Quantum Mechanical, Classical and Semiclassical Treatment. Journal of Physics B: Atomic, Molecular and Optical Physics, 33, 291. https://doi.org/10.1088/0953-4075/33/3/301

[9] Raković, M.J., Uzer, T. and Farrelly, D. (1998) Classical and Quantum Mechanics of an Integrable Limit of the Hydrogen Atom in Combined Circularly Polarized Microwave and Magnetic Fields. Physical Review A, 57, 2814. https://doi.org/10.1103/PhysRevA.57.2814

[10] Ganesan, K. and Lakshmanan, M. (1990) Dynamics of Atomic Hydrogen in a Generalized van der Waals Potential. Physical Review A, 42, 3940-3947.

https://doi.org/10.1103/PhysRevA.42.3940

[11] Ganesan, K. and Lakshmanan, M. (1993) Quantum Chaos of the Hydrogen Atom in a Generalized van der Waals Potential. Physical Review A, 48, 964-976. https://doi.org/10.1103/PhysRevA.48.964

[12] Alhassid, Y., Hinds, E.A. and Meschede, D. (1987) Dynamical Symmetries of the Perturbed Hydrogen Atom: The van der Waals Interaction. Physical Review Letters, 59, 1545-1548. https://doi.org/10.1103/PhysRevLett.59.1545

[13] Paul, W. (1990) Electromagnetic Traps for Charged and Neutral Particles. Reviews of Modern Physics, 62, 531-540. https://doi.org/10.1103/RevModPhys.62.531

[14] Lakshmanan, M. and Sahadevan, R. (1993) Painlevé Analysis, Lie Symmetries, and Integrability of Coupled Nonlinear Oscillators of Polynomial Type. Physics Reports, 224, 1-93. https://doi.org/10.1016/0370-1573(93)90081-N

[15] Klappauf, B.G., Oskay, W.H., Steck, D.A. and Raizen, M.G. (1998) Experimental Study of Quantum Dynamics in a Regime of Classical Anomalous Diffusion. Physical Review Letters, 81, 4044-4047. https://doi.org/10.1103/PhysRevLett.81.4044

[16] Koch, P.M. and van Leeuwen, K.A.H. (1995) The Importance of Resonances in Microwave Ionization of Excited Hydrogen Atoms. Physics Reports, 255, 289-403. https://doi.org/10.1016/0370-1573(94)00093-I

[17] Schmelcher, P. and Schweizer, W. (1998) Atoms and Molecules in Strong External Fields. Plenum Press, New York.

[18] Eleuch, H. and Prasad, A. (2012) Chaos and Regularity in Semiconductor Microcavities. Physics Letters A, 376, 1970-1977. https://doi.org/10.1016/j.physleta.2012.04.050

[19] Potekhin, A.Y. and Turbiner, A.V. (2001) Hydrogen Atom in a Magnetic Field: The Quadripole Moment. Physical Review A: Atomic, Molecular, and Optical Physics, 63, 6. https://doi.org/10.1103/PhysRevA.63.065402

[20] Andreev, A.V., Agam, O., Simons, B.D. and Altshuler, B.L. (1996) Quantum Chaos, Irreversible Classical Dynamics, and Random Matrix Theory. Physical Review Letters, 76, 3947-3950. https://doi.org/10.1103/PhysRevLett.76.3947

[21] Audin, M. (2008) Hamiltonian Systems and Their Integrability. SMF/AMS Text and Monographs, 15, American Mathematical Society.

[22] Kharlamov, M.P., Ryabov, P.E. and Savushkin, A.Y. (2016) Topological Atlas of the Kowalevski-Sokolov Top. Regular and Chaotic Dynamics, 21, 24-65. https://doi.org/10.1134/S1560354716010032

[23] Kharlamov, M.P. (2015) Phase Topology of One System with Separated Variables and Singularities of the Symplectic Structure. Journal of Geometry and Physics, 87, 248-265. https://doi.org/10.1016/j.geomphys.2014.07.004 
[24] Ryabov, P.E. (2014) The Phase Topology of a Special Case of Goryachev Integrability in Rigid Body Dynamics. Sbornik: Mathematics, 205, 1024-1044. https://doi.org/10.1070/SM2014v205n07ABEH004408

[25] Gavrilov, L., Ouazzani-Jamil, M. and Caboz, R. (1993) Bifurcation Diagrams and Fomenko's Surgery on Liouville tori of the Kolossoff Potential. Annales Scientifiques de lÉcole Normale Supérieure, 26, 545-564. https://doi.org/10.24033/asens.1680

[26] Gavrilov, L. (1989) Bifurcations of the Invariant Manifolds in the Generalised Hénon-Heils System. Physica D, 34, 223-239. https://doi.org/10.1016/0167-2789(89)90236-4

[27] Gavrilov, L. (1987) On the Geometry of Goryatchev-Tchaplygin Top. Bulgarian Academy of Sciences, 40, 33-36.

[28] Griffiths, P. and Harris, J. (1978) Principles of Algebraic Geometry. Wiley Interscience, New York.

[29] Fomenko, A.T. (1988) Integrability and Nonintegrability in Geometric and Mechanics. Kluwer Academic Publisher, Heidelberg.

https://doi.org/10.1007/978-94-009-3069-8

[30] Hénon, M. (1982) On the Numerical Computation of Poincaré Maps. Physica D: Nonlinear Phenomena, 5, 412-414. https://doi.org/10.1016/0167-2789(82)90034-3 